\title{
Reconstruction of Deep Posttraumatic Tracheal Stenosis while under Lung Assist
}

\author{
Arpad Pereszlenyi, Stephan Eggeling, Rene-Lars Morgen and Stephan Sklenar
}

Department of Thoracic Surgery, Vivantes Clinics Neukoelln, Berlin, Germany

Correspondence should be addressed to: Arpad Pereszlenyi; arpad.pereszlenyi@vivantes.de

Received 19 May 2013; Accepted 4 July 2013; Published 28 August 2013

Academic Editor: Walter G. Vincken

Copyright (C 2013 Arpad Pereszlenyi, Stephan Eggeling, Rene-Lars Morgen and Stephan Sklenar. Distributed under Creative Commons CC-BY 3.0

\begin{abstract}
Tracheal stenosis is a serious, life-threatening complication with increasing incidence. Among these stenoses, a significant number belongs to the posttraumatic ones. Acute tracheal intervention during an unstable intraoperative condition often requires supporting the respiratory and hemodynamic systems.
\end{abstract}

The present paper is dedicated to this topic; the management of acute posttraumatic tracheal stenosis while applying the support of a lung assist device.

Keywords: Deep Posttraumatic Tracheal Stenosis, Tracheal Reconstruction, Lung Assist Support.

\section{Introduction}

The management of the obstructive tracheal lesion, which develops as a result of long-term intubation, is a complex problem that requires an individual approach. The fully developed stenotic lesion with a high degree of obstruction requires radical surgical resection. The maintenance of adequate ventilation, sufficient oxygenation, as well as carbon dioxide removal is absolutely required during this complex tracheal reconstruction. As is well-known, this could be very challenging especially in case of associated comorbidities of the suffering individual.

We present the case of deep posttraumatic tracheal stenosis that developed as a complication after tracheostomy and long- term intubation during polytrauma management following a severe car accident. For intraoperative respiratory support during the tracheal procedure, the interventional lung assist device (iLA Activve) was used.

\section{Case Description}

A 61 year old male patient with acute inspiratory and expiratory stridor was admitted to our hospital. The CT of the neck and thorax was immediately performed. A deeply located concentric tracheal stenosis ca. $2.5 \mathrm{~cm} \mathrm{-} \mathrm{above} \mathrm{the}$ main carina - was diagnosed. In the CT scan, an aortic stent was also captured. This was performed six months ago due to a severe car accident according to the polytrauma management. In the same time, the tracheostomy and long-term intubation 
were also managed. All this (tracheostomy, aortic stent) can be seen in the CT scans. Fig.1.

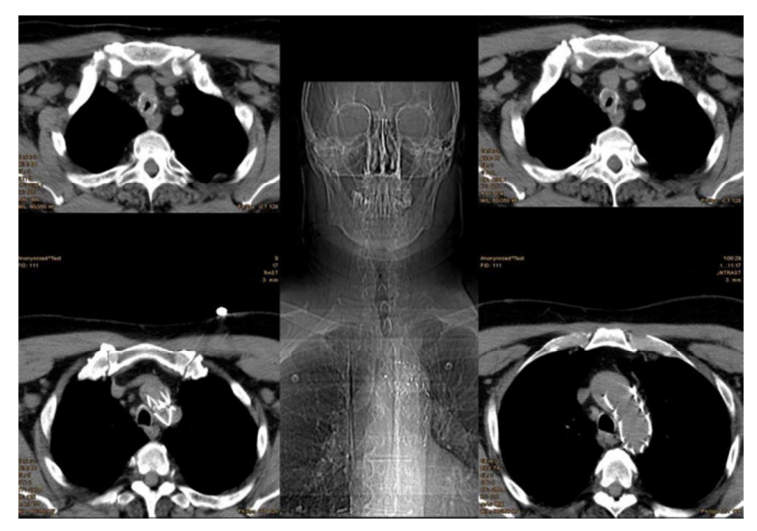

Fig. 1: Images of the Deep Posttraumatic Tracheal Stenosis. The Tracheostomy and Aortic Stent Performed Several Months Ago in Accordance with the Patient's Polytrauma Can be Easily Recognized

An emergency procedure - reconstruction of the trachea - was immediately performed. To support the respiratory and hemodynamic systems during the procedure the lung assist device was chosen. This system contains the iLA Membrane Ventilator, iLA activve pump, and two NovaPort cannulas. For a venovenous lung assist device the cannulation of a jugular and the femoral veins was performed. The iLA Activve, Novalung $\mathrm{GmbH}$, Heilbronn system covers the full range of respiratory support: from highly effective carbon dioxide elimination to complete oxygenation.

The blood drainage is established through the right femoral vein (21 Fr $14 \mathrm{~cm}$ ), and the return of the oxygenated (decarboxylized) blood through the right jugular vein (17 Fr $14 \mathrm{~cm}$ ). The blood is pushed gently and efficiently by the iLA Activve diagonal pump to the iLA Membrane Ventilator where the gas exchange of the artificial lung happens.

The iLA Membrane Ventilator incorporates a heparin-coated hollow-fiber diffusion membrane and removes carbon dioxide effectively and reliably in a very short period of time to achieve the desired target, using low blood flows of approx. $1 \mathrm{~L} / \mathrm{min}$. This extrapulmonary ventilation can be controlled via the sweep gas flow. In cases of respiratory acidosis, the $\mathrm{pH}$ is generally returned to normal physiological levels within a few hours. This can help protect the kidney and other organs and prevent multi-organ failure.

The iLA Activve System was managed with a blood flow of $2.1+0.3 \mathrm{~L} / \mathrm{min}$ and with a gas flow of $5.0+1 \mathrm{~L} / \mathrm{min}$. The patient was normocapnic and normoxemic during the entire procedure (SaO2>95\%). Because it was our first tracheal reconstruction supported by this device, the High Frequency Jet Ventilation was also used. This step was performed immediately after the opening of the trachea below to the stenotic area. Until this moment, quasi static ventilation (PCV, superimposed HFJV, Tidal volume $4 \mathrm{~mL} / \mathrm{kg}$ IBW, Bf $4 / \mathrm{min}$ ) was performed.

Therefore, a segmental tracheal resection with an end-to-end anastomosis was performed. A stenotic segment of 3-4 cm was removed. The anastomosis was constructed by the monofilament absorbable sutures via a single stitches technique, knotting the ties out of the lumen (not inside the lumen of the trachea). No coverage of the anastomosis was performed. The Second Figure shows the preparation of the trachea, and its 
mediastinal release. It is in this way, with the finger of the first surgeon deeply inserted into the mediastinum down to the lung hilus bilateral, that the trachea must be mobilized in order to achieve a tension free anastomosis. Fig. 2.

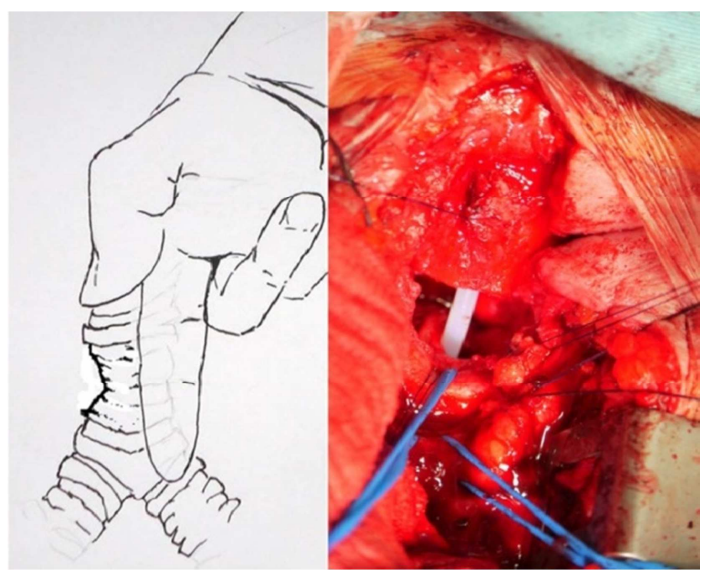

Fig. 2: Scheme of the Deep Tracheal Stenosis with its Mediastinal Release. Intraoperative Photograph Shows the Already Resected Trachea Just before the Reconstruction. The Brachiocephalic Artery and Vein are Mobilized on the Blue Vessel Loops. View through the Sternotomy Approach

The procedure lasts 3 hours, and the iLA Activve support was disconnected immediately after the surgery, together with the extubation of the patient still in the operating theater.

As it stated several times hereinabove, by performing this type of tracheal procedure with this kind of support - iLA Activve System - we experienced very convenient and stable ventilatory and hemodynamic conditions throughout the intervention. There was no need to interrupt the surgery for any kind of adverse event. and end-to-end anastomosis. Any kind of endoscopic management - e.g. laser intervention, airway stenting - was reserved for the case of postoperative complication of restenosis and/or anastomotic insufficiency.

The patient was discharge on the 8th postoperative day with nicely healed tracheal anastomosis (Fig.3). Today - 6 months after the procedure - he is doing well, and carrying out his daily program in full activity.

We decided to perform a primary tracheal reconstruction with segmental resection

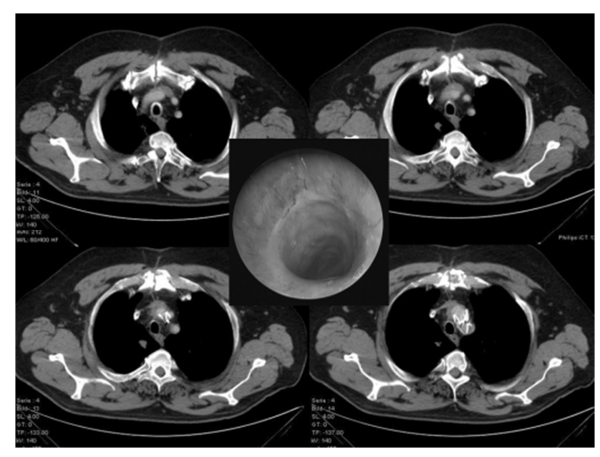

Fig. 3: Postoperative CT Scans of the Reconstructed Trachea. In the Middle, the Endoscopic View of the Nicely Healed Tracheal Anastomosis Can be Seen 


\section{Discussion}

We report our novel experience with the iLA Activve System (Novalung $\mathrm{GmbH}$, Heilbronn) during the tracheal reconstruction for the deep posttraumatic tracheal stenosis.

The use of Novalung has been reported in many cases worldwide, including lung infection, adult respiratory distress syndrome, trauma, and others. Matheis (2003); von Mach et al (2006); Bein et al (2005).

Probably the widest indication spectrum has Novalung in the lung transplantation medicine as a bridge to definitive surgery with its prolonged postoperative support. Jackson et al (2008); Fischer et al (2006); Bartosik et al (2011).

It is a pumpless variant - formerly known as PECLA (pumpless extracorporeal lung assist) - and is also well known in intensive care medicine for the treatment of acute lung failure. Bein et al (2005).

According to our knowledge, the use of iLA for the reconstruction of the deep posttraumatic tracheal stenosis is the first one reported in the literature.

As illustrated by our case, the advantage of iLA Activve System is that the hemodynamically (and respiratory) unstable intra- and perioperative course can be fully controlled. Moreover, due to the clinical condition and due to the technical and anatomical difficulties, the definitive resection and reconstruction is very challenging by standard intubation and ventilation protocols. Under these circumstances, the iLA Acctive System offers a unique service to overcome this demanding intra- and postoperative period. By performing tracheal reconstruction by iLA support we experienced a very convenient and stable intraoperative situation throughout the intervention. There was no need to interrupt the surgery for any kind of adverse event. Finally, all of the above mentioned resulted into the early extubation (and decannulation) of the patient with immediate spontaneous breathing and coughing activity, which (as is known from the tracheal surgery) is extremely important for the further postoperative course of the patient. The patient made a complete recovery after the surgery and was discharged on the 8th postoperative day.

\section{Abbreviations}

iLA - interventional lung assist Novalung

SaO2 - arterial oxygenation saturation

HFJV - high frequency jet ventilation

PCV - pressure controlled ventilation

IBW - ideal body weight

$\mathrm{Bf}$ - breathing frequency

PECLA - pumpless extracorporeal lung assist

\section{References}

Bartosik, W., Egan, J. J. \& Wood, A. E. (2011). "The Novalung Interventional Lung Assist as Bridge to Lung Transplantation for Self-Ventilating Patients - Initial Experience," Interactive CardioVasc Thoracic Surgery, 13 198-200.

Bein, T., Scherer, M. N., Philipp, A., Weber, F. \& Woertgen, C. (2005). "Pumpless Extracorporeal Lung Assist (Pecla) In Patients with Acute Respiratory Distress Syndrome and Severe Brain Injury," Journal of Trauma-Injury Infection \& Critical Care, 58 1294-1297.

Fischer, S., Simon, A. R., Welte, T., Hoeper, M. M., Meyer, A., Tessmann, R., Gohrbandt, B., Gottlieb, J., Haverich, A. \& Strueber, M. (2006). "Bridge to Lung Transplantation with the Novel Pumpless Interventional Lung Assist Device Novalung," Interactive CardioVasc Thoracic Surgery, 131 (3) 719723.

Jackson, A., Cropper, J., Pye, R., Junius, F., Malouf, M. \& Glanville, A. (2008). "Use of Extracorporeal Membrane Oxygenation as 
a Bridge to Primary Lung Transplant: 3 Consecutive, Successful Cases and a Review of the Literature," The Journal of Heart and Lung Transplantation, 27 (3) 348-352.

Matheis, G. (2003). "New Technologies for Respiratory Assist," Perfusion, 18 (1) 245251.

Von Mach, M. A., Kaes, J., Omogbehin, B., Sagoschen, I., Wiechelt, J., Kaiser, K., Sauer, O. \& Weilemann, L. S. (2006). "An Update on Interventional Lung Assist Devices and Their Role in Acute Respiratory Distress Syndrome," Lung, 184 (3) 169-175. 